Research Article

\title{
A Study of Fractional Differential Equation with a Positive Constant Coefficient via Hilfer Fractional Derivative
}

\author{
Hoa Ngo Van $\mathbb{1}^{1,2}$ and Vu Ho $\mathbb{i}^{3,4}$ \\ ${ }^{1}$ Division of Computational Mathematics and Engineering, Institute for Computational Science, Ton Duc Thang University, \\ Ho Chi Minh, Vietnam \\ ${ }^{2}$ Faculty of Mathematics and Statistics, Ton Duc Thang University, Ho Chi Minh, Vietnam \\ ${ }^{3}$ Institute of Fundamental and Applied Sciences, Duy Tan University, Ho Chi Minh 550000, Vietnam \\ ${ }^{4}$ Faculty of Natural Sciences, Duy Tan University, Da Nang 550000, Vietnam \\ Correspondence should be addressed to Vu Ho; hovu1@duytan.edu.vn
}

Received 1 April 2020; Accepted 16 June 2020; Published 14 August 2020

Guest Editor: Shilpi Jain

Copyright ( 92020 Hoa Ngo Van and Vu Ho. This is an open access article distributed under the Creative Commons Attribution License, which permits unrestricted use, distribution, and reproduction in any medium, provided the original work is properly cited.

The aim of the paper is to consider the existence and uniqueness of solution of the fractional differential equation with a positive constant coefficient under Hilfer fractional derivative by using the fixed-point theorem. We also prove the bounded and continuous dependence on the initial conditions of solution. Besides, Hyers-Ulam stability and Hyers-Ulam-Rassias stability are discussed. Finally, we provide an example to demonstrate our main results.

\section{Introduction}

In recent years, the study of the fractional differential and integral equation (FDE and IDE for short) has become the topic of the applied mathematics. FDE and IDE have been used as a tool mathematical to the modeling of many phenomena in various fields for example, in theory of signal processing, physics, economics, and chaotic dynamics. The reader can refer to the books (see Varsha [1], Kilbas et al. [2], Miller et al. [3], Abbas et al. [4], and Zhou et al. [5]) or the papers (see Zin et al. [6], Ma et al. [7], P. Agarwal et al. [8, 9], Rameshet al. [10], Vivek et al. [11], O’Regan et al. [12], and Duc et al. [13]).

Tate and Dinde [14] proved the existence of solution of the problem:

$$
{ }^{c} \mathfrak{D}_{0^{+}}^{\alpha} \xi(t)=\lambda \xi(t)+\zeta(t, \xi(t)), \quad \xi(0)=x_{0} \in \mathfrak{R}, \lambda>0,
$$

where the symbol ${ }^{C} \mathfrak{D}_{0^{+}}^{\alpha}$ is Caputo fractional derivative and $\zeta \in C([0, a], \Re)$. Besides, the authors also considered the properties of solutions of this problem such as the boundedness of solution and the continuous dependence of solutions on the initial conditions. In [15], Tate et al. also performed the same study as [14] for the class of fractional integro-differential equations with positive constant coefficient.

In 2018, Sousa and Oliveira [16] have introduced a new fractional derivative with respect to another function the socalled $\psi$-Hilfer fractional derivative, and the properties of this concept were also presented. Besides, the authors considered the relationship between the $\psi$-Hilfer fractional derivative and the other fractional derivative such as Riemann-Liouville fractional derivative, Caputo fractional derivative, Hadamard fractional derivative, Katugampola fractional derivative, and Chen fractional derivative. By using the $\psi$-Hilfer fractional derivative, Sousa and Oliveira [17] studied the existence and uniqueness of solution of the initial valued problem for FDEs. The continuous dependence of solution on the initial condition was also considered. The stability theory for FDIs and IDEs via $\psi$-Hilfer fractional derivative have also been discussed (see [18-22]). In [23], by using Gronwall inequality and Picard operator theory, Kharade and Kucche proved the existence and uniqueness of solutions for impulsive implicit delay 
$\psi$-Hilfer fractional differential equations. The Ulam-Hyers-Mittag-Leffler stability was also considered.

Motivated by Tate el al. [14, 15], Sousa et al. [16], and Kharade et al. [23], in this paper, we investigate the existence and uniqueness of solutions and some properties of solutions of the following fractional differential equation with the constant coefficient $\lambda>0$ :

$$
{ }_{0^{+}}^{\mathbb{A}} \mathscr{D}^{h, v ; g} \xi(t)=\lambda \xi(t)+\zeta(t, \xi(t)),
$$

with the initial condition

$$
\mathfrak{\Im}_{0^{+}}^{1-\gamma, g} \xi(0)=A,
$$

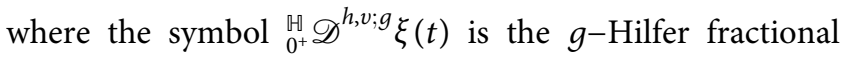
derivative of $\xi$ with $0<h<1,0 \leq v \leq 1$ and $\xi:[0, a] \longrightarrow \mathfrak{R}$ is a continuous function, $\zeta$ is a continuous function with respect to $t$ and $\xi$ on $[0, a] \times \mathfrak{R}, \mathfrak{\Im}_{0^{+}}^{1-\gamma, g}(\cdot)$ is $g$-RiemannLiouville fractional integral with $0 \leq \gamma=h+v(1-h)<1$, and $A$ is a given constant.

\section{Preliminaries}

In this section, we introduce some notations and some concepts which are used throughout this paper. This result can be found in the books $[3,8]$ and the papers $[16,17]$.

Let $C([0, a], \Re)$ be the space of all continuous functions $\zeta:[0, a] \longrightarrow \mathfrak{R}$ and $C^{n}([0, a], \mathfrak{R})$ be the space of all $n$-times continuously differentiable functions on $[0, a]$. We will introduce the weighted spaces of all continuous functions $C_{\gamma, g}([0, a]):=\left\{\zeta:(0, a] \longrightarrow \mathfrak{R}:(g(t)-g(0))^{\gamma} \zeta(t) \in C([0, a])\right\}$,

with the norm

$$
\begin{aligned}
\|\zeta\|_{C_{\gamma, g}([0, a])} & =\left\|(g(t)-g(0))^{\gamma} \zeta(t)\right\|_{C([0, a])} \\
& =\max _{t \in[0, a]}\left|(g(t)-g(0))^{\gamma} \zeta(t)\right| \\
C_{1-\gamma, g}([0, a]) & :=\left\{\zeta:(0, a] \longrightarrow \Re:(g(t)-g(0))^{1-\gamma} \zeta(t)\right. \\
& \in C([0, a])\},
\end{aligned}
$$

with the norm

$$
\begin{aligned}
\|\zeta\|_{C_{1-\gamma, g}([0, a])} & =\left\|(g(t)-g(0))^{1-\gamma} \zeta(t)\right\|_{C([0, a])} \\
& =\max _{t \in[0, a]}\left|(g(t)-g(0))^{1-\gamma} \zeta(t)\right| .
\end{aligned}
$$

Let function $f$ be integrable on $[0, a]$ and function $g:[0, a] \longrightarrow \mathfrak{R}_{+}$be increasing on $[0, a]$ with $g^{\prime}(t) \neq 0$ for all $t \in[0, a]$. Then, the $g$-Riemann-Liouville fractional integral of $f$ with respect to $g$ is defined by

$$
\mathfrak{J}_{0^{+}}^{h ; g} \xi(t)=\frac{1}{\Gamma(h)} \int_{0}^{t} \mathfrak{Q}_{g}^{h}(t, s) \xi(s) \mathrm{d} s, \quad h>0,
$$

where $\mathfrak{Q}_{g}^{h}(t, s)=g^{\prime}(s)(g(t)-g(s))^{h-1}$ for all $t, s \in[0, a]$.
Let $n-1<h<n, n \in \mathbb{N}$, and $f \in C^{n}([0, a], \mathfrak{R})$. Then, the Hilfer fractional derivative ${ }^{H} \mathfrak{D}_{0^{+}}^{h, v ; g} \xi(\cdot)$ of the function $\xi$ of order $h$ and $v \in[0,1]$ is given by

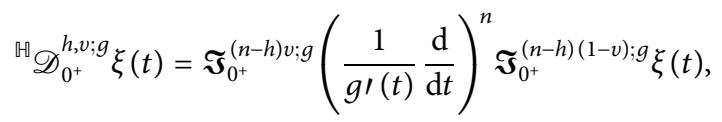

where $n=[h]+1$.

Theorem 1 (see $[16,17])$. If $\zeta \in C^{1}([0, a], \Re), h>0$, and $v \in[0,1]$, then

$$
\begin{aligned}
& \text { (i) }{ }^{\mathbb{N}} \mathscr{D}_{0^{+}}^{h, v g} \mathfrak{\Im}_{0^{+}}^{h ; g} \xi(t)=\xi(t) \text { and } \forall t \in[0, a] \\
& \text { (ii) } \mathfrak{J}_{0^{+}}^{\text {; }} \mathfrak{D}_{0^{+}}^{h, v ; g} \xi(t)=\xi(t)-\sum_{k=0}^{n}(g(t)-g(0))^{\gamma-k} / \\
& \Gamma(\gamma-k+1) \mathfrak{J}_{0^{+}}^{(1-h)}(1-v) ; g \\
& \Gamma(0) \text { and } \gamma=h+v(1-h)
\end{aligned}
$$

Lemma 1 (see [17]). Let $a(t)$ and $b(t)$ be integrable functions and $c \in C([0, a], \mathfrak{R})$. Let $g \in C^{1}([0, a], \mathfrak{R})$ such that $g^{\prime}(t)>0$ for any $t \in[0, a]$. Assume that $a(t)$ and $b(t)$ are nonnegative and $g$ is nonnegative and nondecreasing. If

$$
a(t) \leq b(t)+c(t) \int_{0}^{t} \mathfrak{Q}_{g}^{h}(t, s) a(s) \mathrm{d} s,
$$

then

$$
a(t) \leq b(t) \mathbb{E}_{h}\left(c(t) \Gamma(h)(g(t)-g(0))^{h}\right), \quad \forall t \in[0, a],
$$

where $\mathbb{E}_{h}(\xi)$ is Mittag-Leffler function is defined by

$$
\mathbb{E}_{h}(\xi)=\sum_{k=0}^{\infty} \frac{\xi^{k}}{\Gamma(1+k h)}, \quad h>0, k \in \mathbb{N} .
$$

Definition 1 (see $[21,23])$. Problem (2) is called Hyers-Ulam stable if there exists a positive constant $C_{\zeta}$ such that, for any $\varepsilon>0$ and for each $\xi \in C_{1-\gamma, g}([0, a])$ satisfying the inequality,

$$
\left|\begin{array}{l}
\mathbb{\sharp} \\
0^{+}
\end{array} \mathscr{D}^{h, v ; g} \xi(t)-\lambda \xi(t)-\zeta(t, \xi(t))\right| \leq \varepsilon, \quad \forall t \in[0, a],
$$

there exists a solution $\widehat{\xi}_{\in} C_{1-\gamma, g}([0, a])$ of problem (2) satisfying

$$
|\xi(t)-\widehat{\xi}(t)| \leq C_{\zeta} \varepsilon, \quad \forall t \in[0, a]
$$

Definition 2 (see [21, 23]). Problem (2) is called Hyers-Ulam-Rassias stable, with respect to $\phi \in C([0, a], \mathfrak{R})$, if there exists a positive constant $C_{\zeta, \phi}$ such that, for any $\varepsilon>0$ and for each $\xi \in C_{1-\gamma, g}([0, a])$ satisfying the inequality,

$$
\left|\mathbb{0}^{+} \mathscr{D}^{h, v ; g} \xi(t)-\lambda \xi(t)-\zeta(t, \xi(t))\right| \leq \epsilon \phi(t), \quad \forall t \in[0, a],
$$

there exists a solution $\widehat{\xi}_{\in} C_{1-\gamma, g}([0, a])$ of problem (2) satisfying

$$
|\xi(t)-\widehat{\xi}(t)| \leq C_{\zeta, \phi} \in \phi(t), \quad \forall t \in[0, a] .
$$




\section{Main Results}

Firstly, we note that applying the fractional integral operator $\mathfrak{\Im}_{0^{+}}^{h ; g}(\cdot)$ to both sides of equation (2), we obtain

$\mathfrak{\Im}_{0^{+} 0^{+}}^{h ; g \mathbb{}} \mathfrak{D}^{h, v ; g} \xi(t)=\lambda \mathfrak{\Im}_{0^{+}}^{h ; g} \xi(t)+\mathfrak{\Im}_{0^{+}}^{h ; g} \zeta(t, \xi(t)), \quad \forall t \in[0, a]$.

Using Theorem 1 and the initial condition (3), we have the following integral equation:

$$
\begin{aligned}
\xi(t)= & \frac{(g(t)-g(0))^{\gamma-1}}{\Gamma(\gamma)} \mathfrak{J}_{0^{+}}^{h ; g} \xi(0)+\lambda \mathfrak{J}_{0^{+}}^{h ; g} \xi(t) \\
& +\mathfrak{J}_{0^{+}}^{(1-h)(1-v) ; g} \zeta(t, \xi(t)) \\
= & \frac{(g(t)-g(0))^{\gamma-1}}{\Gamma(\gamma)} A+\frac{\lambda}{\Gamma(h)} \int_{0}^{t} \mathfrak{Q}_{g}^{h}(t, s) \xi(s) \mathrm{d} s \\
& +\frac{1}{\Gamma(h)} \int_{0}^{t} \mathfrak{Q}_{g}^{h}(t, s) \zeta(s, \xi(s)) \mathrm{d} s,
\end{aligned}
$$

for any $t \in[0, a]$.

On the contrary, if $\xi$ satisfies equation (17), then $\xi$ satisfies equations (2 and 3. Moreover, operating the fractional derivative operator ${ }_{0^{+}}^{\mathbb{H}} \mathfrak{D}^{h, v ; g}(\cdot)$ on both sides of equation (17), we obtain

$$
\begin{aligned}
& { }_{0^{+}}^{\mathbb{W}} \mathscr{D}^{h, v ; g} \xi(t)={ }_{0^{+}}^{\mathbb{H}} \mathfrak{D}^{h, v ; g}\left(\frac{(g(t)-g(0))^{\gamma-1}}{\Gamma(\gamma)} \mathfrak{J}_{0^{+}}^{h ; g} \xi(0)\right) \\
& +{ }_{0^{+}}^{\mathbb{\sharp}} \mathfrak{D}^{h, v ; \mathfrak{g}}\left(\lambda \mathfrak{\Im}_{0^{+}}^{h ; g} \xi(t)\right)+{ }_{0^{+}}^{\mathbb{\sharp}} \mathfrak{D}^{h, v ; \mathfrak{g}} \\
& \cdot\left(\mathfrak{\Im}_{0^{+}}^{(1-h)(1-v) ; g} \zeta(t, \xi(t))\right), \quad \forall t \in[0, a] .
\end{aligned}
$$

By Theorem 1, we have

$$
{ }_{0^{+}}^{\mathbb{N}} \mathscr{D}^{h, v ; g}\left(\frac{(g(t)-g(0))^{h-1}}{\Gamma(\gamma)} \mathfrak{\Im}_{0^{+}}^{h ; g} \xi(0)\right)=0, \quad 0<h<1 .
$$

Combining (18) and (19), we imply

$$
{\stackrel{\mathbb{N}}{0^{+}}}^{\mathbb{N}} \mathscr{D}^{h, v ; g} \xi(t)=\lambda \xi(t)+\zeta(t, \xi(t)), \quad \forall t \in[0, a] .
$$

Next, we verify that the initial condition 3 holds. Indeed, applying the Riemann-Liouville fractional integral $\mathfrak{\Im}_{0^{+}}^{1-v}(\cdot)$ on both sides of equation (17), we have

$$
\begin{aligned}
\mathfrak{\Im}_{0^{+}}^{1-v} \xi(t)= & \mathfrak{\Im}_{0^{+}}^{1-v}\left(\frac{(g(t)-g(0))^{\gamma-1}}{\Gamma(\gamma)} \mathfrak{\Im}_{0^{+}}^{h ; g} \xi(0)\right) \\
& +\mathfrak{\Im}_{0^{+}}^{1-v}\left(\lambda \boldsymbol{\Im}_{0^{+}}^{h ; g} \xi(t)\right)+\mathfrak{\Im}_{0^{+}}^{1-v}\left(\mathfrak{\Im}_{0^{+}}^{(1-h)(1-v) ; g} \zeta(t, \xi(t))\right), \quad t \in[0, a] .
\end{aligned}
$$

Taking $t \longrightarrow 0$ in equation $(21)$, we have

$$
\mathfrak{\Im}_{0^{+}}^{1-v} \xi(t)=A
$$

In summary, we can conclude that $\xi$ satisfies equations ( 2 and 3 if and only if $\xi$ satisfies the following integral equation:

$$
\begin{aligned}
\xi(t)= & \frac{(g(t)-g(0))^{\gamma-1}}{\Gamma(\gamma)} A+\frac{\lambda}{\Gamma(h)} \int_{0}^{t} \mathfrak{Q}_{g}^{h}(t, s) \xi(s) \mathrm{d} s \\
& +\frac{1}{\Gamma(h)} \int_{0}^{t} \mathfrak{Q}_{g}^{h}(t, s) \zeta(s, \xi(s)) \mathrm{d} s,
\end{aligned}
$$

for any $t \in[0, a]$.

3.1. Existence and Uniqueness Solution for Problem (2). In this section, we will prove the existence and uniqueness solution of equation (2) with the initial condition (3). Firstly, we assume that the function $\zeta$ satisfies the following assumption: there exists a constant $L>0$ such that

$$
(\mathrm{H} 1)\left|\zeta\left(t, \xi_{1}\right)-\zeta\left(t, \xi_{2}\right)\right| \leq L\left|\xi_{1}-\xi_{2}\right|, \quad t \in[0, a]
$$

Theorem 2. Assume that assumption (H1) is satisfied. Then, problems (2) and (3) have at least one solution

Proof. We set $\widetilde{\zeta}:=\zeta(t, 0)$ for any $t \in[0, a]$. Let us define the set

$$
\mathcal{U}_{r}:=\left\{\xi \in C_{1-\gamma ; g}([0, a]):\|\xi\|_{C_{1-\gamma ; g}} \leq r\right\},
$$

with

$$
\begin{aligned}
r:= & \frac{|A|}{\Gamma(\gamma)}+\frac{B(h, \gamma)(g(a)-g(0))^{h}}{\Gamma(h)}(\lambda+L)\|\xi\|_{C_{1-\gamma ; g}} \\
& +\frac{B(h, \gamma)(g(a)-g(0))^{h}}{\Gamma(h)}\|\widetilde{\zeta}\|_{C_{1-\gamma ; g}}, \quad \forall t \in[0, a] .
\end{aligned}
$$

It is easy to see that $\mathcal{U}_{r}$ is a nonempty, closed, bounded, and convex subset of Banach space $C_{1-\gamma, g}([0, a])$.

Consider the operator $\mathbb{K}: \mathscr{U}_{r} \longrightarrow \mathcal{U}_{r}$ given by

$$
\begin{aligned}
(\mathbb{K} \xi)(t)= & \frac{(g(t)-g(0))^{\gamma-1}}{\Gamma(h)} A+\frac{\lambda}{\Gamma(h)} \int_{0}^{t} \mathfrak{Q}_{g}^{h}(t, s) \xi(s) \mathrm{d} s \\
& +\frac{1}{\Gamma(h)} \int_{0}^{t} \mathfrak{Q}_{g}^{h}(t, s) \zeta(s, \xi(s)) \mathrm{d} s,
\end{aligned}
$$

for any $t \in[0, a]$.

Firstly, we prove that the fixed point of the operator $\mathbb{K}$ is a solution of equations (2) and (3). For any $\xi \in C_{1-\gamma ; g}([0, a])$ and for each $t \in[0, a]$, we have the following estimate: 


$$
\begin{aligned}
\left|(\mathbb{K} \xi)(t)(g(t)-g(0))^{1-\gamma}\right|= & \left|\frac{A}{\Gamma(\gamma)}+\frac{\lambda(g(t)-g(0))^{1-\gamma}}{\Gamma(h)} \int_{0}^{t} \mathfrak{Q}_{g}^{h}(t, s) \xi(s) \mathrm{d} s+\frac{(g(t)-g(0))^{1-\gamma}}{\Gamma(h)} \int_{0}^{t} \mathfrak{Q}_{g}^{h}(t, s) \zeta(s, \xi(s)) \mathrm{d} s\right| \\
\leq & \frac{|A|}{\Gamma(\gamma)}+\frac{\lambda(g(t)-g(0))^{1-\gamma}}{\Gamma(h)} \int_{0}^{t} \mathfrak{Q}_{g}^{h}(t, s)|\xi(s)| \mathrm{d} s+\frac{(g(t)-g(0))^{1-\gamma}}{\Gamma(h)} \int_{0}^{t} \mathfrak{Q}_{g}^{h}(t, s)|\zeta(s, \xi(s))| \mathrm{d} s \\
\leq & \left|\frac{|A|}{\Gamma(\gamma)}+\frac{\lambda(g(t)-g(0))^{1-\gamma}}{\Gamma(h)} \int_{0}^{t} \mathfrak{Q}_{g}^{h}(t, s)\right| \xi(s)\left|\mathrm{d} s+\frac{(g(t)-g(0))^{1-\gamma}}{\Gamma(h)} \int_{0}^{t} \mathfrak{Q}_{g}^{h}(t, s)\right| \zeta(s, \xi(s)) \\
& -\zeta(s, 0)+\zeta(s, 0) \mid \mathrm{d} s \\
\leq & \frac{|A|}{\Gamma(\gamma)}+\frac{\lambda(g(t)-g(0))^{1-\gamma}}{\Gamma(h)} B(h, \gamma)(g(t)-g(0))^{h+\gamma-1}\|\xi\|_{C_{1-\gamma ; g}} \\
& +\frac{(g(t)-g(0))^{1-\gamma}}{\Gamma(h)} B(h, \gamma)(g(t)-g(0))^{h+\gamma-1} L\|\xi\|_{C_{1-\gamma ; g}} \\
& +\frac{(g(t)-g(0))^{1-\gamma}}{\Gamma(h)} B(h, \gamma)(g(t)-g(0))^{h+\gamma-1}\|\widetilde{\zeta}\|_{C_{1-\gamma ; g}},
\end{aligned}
$$

for any $t \in[0, a]$.

Combining the estimation above with the definition of $\mathcal{U}_{r}$, we infer that

$$
\begin{gathered}
\left|(\mathbb{K} \xi)(t)(g(t)-g(0))^{1-\gamma}\right| \leq \frac{|A|}{\Gamma(\gamma)}+\frac{B(h, \gamma)(g(a)-g(0))^{h}}{\Gamma(h)} \\
\cdot(\lambda+L)\|\xi\|_{C_{1-\gamma ; g}}+\frac{B(h, \gamma)(g(a)-g(0))^{h}}{\Gamma(h)}\|\tilde{\xi}\|_{C_{1-\gamma ; g}}=r .
\end{gathered}
$$

Hence, we conclude the operator $\mathbb{K}$ maps into itself.

Secondly, $\mathbb{K}\left(\mathscr{U}_{r}\right)$ is uniformly bounded since $\mathbb{K}\left(\mathcal{U}_{r}\right) \subset \mathcal{U}_{r}$.

Thirdly, we will prove that the operator $\mathbb{K}$ is continuous. Let the sequence $\left\{\xi_{n}\right\} \in \mathscr{U}_{r}$ and $\xi \in \mathscr{U}_{r}$ such that

$$
\left\|\xi_{n}-\xi\right\|_{C_{1-\gamma ; g}} \longrightarrow 0, \quad \text { as } n \longrightarrow \infty \text {. }
$$

For any $t \in[0, a]$, we have the following estimate:

$$
\begin{aligned}
\left|\left(\left(\mathbb{K} \xi_{n}\right)(t)-(\mathbb{K} \xi)(t)\right)(g(t)-g(0))^{1-\gamma}\right| \leq & \frac{\lambda(g(t)-g(0))^{1-\gamma}}{\Gamma(h)} \int_{0}^{t} \mathfrak{Q}_{g}^{h}(t, s)\left|\xi_{n}(s)-\xi(s)\right| \mathrm{d} s \\
& +\frac{(g(t)-g(0))^{1-\gamma}}{\Gamma(h)} \int_{0}^{t} \mathfrak{Q}_{g}^{h}(t, s)\left|\zeta\left(s, \xi_{n}(s)\right)-\zeta(s, \xi(s))\right| \mathrm{d} s \\
\leq & \frac{B(h, \gamma)}{\Gamma(h)}(g(t)-g(0))^{h} \lambda\left\|\xi_{n}(\cdot)-\xi(\cdot)\right\|_{C_{1-\gamma ; g}} \\
& +\frac{B(h, \gamma)}{\Gamma(h)}(g(t)-g(0))^{h}\left\|\zeta\left(\cdot, \xi_{n}(\cdot)\right)-\zeta(\cdot, \xi(\cdot))\right\|_{C_{1-\gamma ; g}, \quad \forall t \in[0, a] .}
\end{aligned}
$$

Hence, for any $t \in[0, a]$, we have

$$
\begin{aligned}
\left\|\mathbb{K} \xi_{n}-\mathbb{K} \xi\right\|_{C_{1-\gamma ; g} \leq} & \frac{B(h, \gamma)}{\Gamma(h)}(g(a)-g(0))^{h} \lambda\left\|\xi_{n}(\cdot)-\xi(\cdot)\right\|_{C_{1-\gamma ; g}} \\
& +\frac{B(h, \gamma)}{\Gamma(h)}(g(a)-g(0))^{h} \\
& \cdot\left\|\zeta\left(\cdot, \xi_{n}(\cdot)\right)-\zeta(\cdot, \xi(\cdot))\right\|_{C_{1-\gamma ; g}}
\end{aligned}
$$

Based on the continuity of function $\zeta$, we obtain

$\left\|\zeta\left(\cdot, \xi_{n}(\cdot)\right)-\zeta(\cdot, \xi(\cdot))\right\|_{C_{1-\gamma: g}} \longrightarrow 0, \quad$ as $n \longrightarrow \infty$.

So, for any $t \in[0, a]$, we obtain

$$
\left\|\mathbb{K} \xi_{n}-\mathbb{K} \xi\right\|_{C_{1-\gamma ; g}} \longrightarrow 0, \quad \text { as } n \longrightarrow \infty,
$$

which leads to operator $\mathbb{K}$ which is continuous. 
Next, we will show that $\mathbb{K}\left(\mathcal{U}_{r}\right)$ is equicontinuous. Let $t_{1}, t_{2} \in[0, a]$ such that $0<t_{1}<t_{2}<a$ and $\xi \in \mathscr{U}_{r}$, and we have

$$
\begin{aligned}
\left|(\mathbb{K} \xi)\left(t_{2}\right)\left(g\left(t_{2}\right)-g(0)\right)^{1-\gamma}-(\mathbb{K} \xi)\left(t_{1}\right)\left(g\left(t_{1}\right)-g(0)\right)^{1-\gamma}\right| \\
=\mid \frac{\lambda\left(g\left(t_{2}\right)-g(0)\right)^{1-\gamma}}{\Gamma(h)} \int_{0}^{t_{2}} \mathfrak{Q}_{g}^{h}\left(t_{2}, s\right) \xi(s) \mathrm{d} s+\frac{\left(g\left(t_{2}\right)-g(0)\right)^{1-\gamma}}{\Gamma(h)} \int_{0}^{t_{2}} \mathfrak{Q}_{g}^{h}\left(t_{2}, s\right) \zeta(s, \xi(s)) \mathrm{d} s \\
\quad-\frac{\lambda\left(g\left(t_{1}\right)-g(0)\right)^{1-\gamma}}{\Gamma(h)} \int_{0}^{t_{1}} \mathfrak{Q}_{g}^{h}\left(t_{1}, s\right) \xi(s) \mathrm{d} s-\frac{\left(g\left(t_{1}\right)-g(0)\right)^{1-\gamma}}{\Gamma(h)} \int_{0}^{t_{1}} \mathfrak{Q}_{g}^{h}\left(t_{1}, s\right) \zeta(s, \xi(s)) \mathrm{d} s \mid \\
\quad \leq \frac{1}{\Gamma(h)} B(h, \gamma)\left(\lambda\|\xi\|_{C_{1-\gamma ; g}}+\|\zeta\|_{C_{1-\gamma ; g}}\right) \mid\left(g\left(t_{2}\right)-g(0)\right)^{h}-\left(g\left(t_{1}\right)-g(0)\right)^{h} .
\end{aligned}
$$

As $t_{2} \longrightarrow t_{1}$, we imply the right-hand side of the estimation above tend to 0 , that is, $\mathbb{K}\left(\mathcal{U}_{r}\right)$ is equicontinuous.

Finally, we see that all conditions of Schauder fixed point theorem are satisfied. So, we can conclude that problem (2) and (3) has at least one solution. The proof is complete.

Theorem 3. Let $\tilde{a} \in(0, a)$. Assume that assumption (H1) is satisfied. If

$$
\frac{B(h, \gamma)(g(\widetilde{a})-g(0))^{h}(\lambda+L)}{\Gamma(h)}<1,
$$

then problems (2) and (3) have a unique solution on $[0, \tilde{a}]$.

Proof. To prove this theorem, divide the proof into two steps. Now, we define the following set:

$$
\mathcal{U}_{\rho}:=\left\{\xi \in C_{1-\gamma, g}([0, \tilde{a}]):\|\xi\|_{C_{1-\gamma, g}([0, \tilde{a}])} \leq \rho\right\},
$$

where

$$
\rho:=\frac{A \Gamma(h)}{\Gamma(\gamma)\left[\Gamma(h)-(1+\lambda+L)(g(\widetilde{a})-g(0))^{h}\right]} .
$$

Let us define the operator $\mathscr{B}: C_{1-\gamma, g}([0, \tilde{a}]) \longrightarrow$ $C_{1-\gamma, g}([0, \tilde{a}])$ as follows:

$$
\begin{aligned}
(\mathscr{B} \xi)(t)= & \frac{(g(t)-g(0))^{\gamma-1}}{\Gamma(\gamma)} A+\frac{\lambda}{\Gamma(h)} \int_{0}^{t} \mathfrak{Q}_{g}^{h}(t, s) \xi(s) \mathrm{d} s \\
& +\frac{1}{\Gamma(h)} \int_{0}^{t} \mathfrak{Q}_{g}^{h}(t, s) \zeta(s, \xi(s)) \mathrm{d} s, \quad \forall t \in[0, \tilde{a}] .
\end{aligned}
$$

Step 1: similarl to the proof of Theorem 2, we can infer that the functions of $\mathscr{B} \mathcal{U}_{\rho}$ are uniformly bounded in $C_{1-\gamma, g}([0, \tilde{a}])$.

Step 2: we will show that $\mathscr{B}$ is a contraction on $C_{1-\gamma, g}([0, \tilde{a}])$. For any $t \in[0, \tilde{a}]$ and $\xi_{1}, \xi_{2} \in C_{1-\gamma, g}([0, \tilde{a}])$, we have

$$
\begin{aligned}
\left|\left(\left(\mathbb{K} \xi_{1}\right)(t)-\left(\mathbb{K} \xi_{2}\right)(t)\right)(g(t)-g(0))^{1-\gamma}\right| \leq & \frac{\lambda(g(t)-g(0))^{1-\gamma}}{\Gamma(h)} \int_{0}^{t} \mathfrak{Q}_{g}^{h}(t, s)\left|\xi_{1}(s)-\xi_{2}(s)\right| \mathrm{d} s \\
& +\frac{(g(t)-g(0))^{1-\gamma}}{\Gamma(h)} \int_{0}^{t} \mathfrak{Q}_{g}^{h}(t, s)\left|\zeta\left(s, \xi_{1}(s)\right)-\zeta\left(s, \xi_{2}(s)\right)\right| \mathrm{d} s \\
& \leq \frac{B(h, \gamma)}{\Gamma(h)}(g(t)-g(0))^{h}(\lambda+L)\left\|\xi_{1}-\xi_{2}\right\|_{C_{1-\gamma, g}([0, \tilde{a}])^{*}}
\end{aligned}
$$

Combining the estimation above with assumption (36), we obtain

$$
\left\|\mathbb{K} \xi_{1}-\mathbb{K} \xi_{2}\right\|_{C_{1-\gamma, g}([0, \tilde{a}])} \leq\left\|\xi_{1}-\xi_{2}\right\|_{C_{1-\gamma, g}([0, \tilde{a}])},
$$

that is, $\mathscr{B}$ is a contraction on $C_{1-\gamma, g}([0, \tilde{a}])$.

Here, we see that all conditions in the Banach fixed point theorem are satisfied. Therefore, there exists a unique solution of problems (2) and (3). This proof is completed.
3.2. Continuous Dependence and Boundedness of Solution of Problem (2). In this section, we will study the continuous dependence of solutions on initial conditions and the boundedness of solution of equations (2) and (3). Now, we consider the following problems:

$$
\begin{aligned}
& {\stackrel{\mathbb{N}}{0^{+}}}_{\mathfrak{D}^{h, v ; g}}^{\mathbb{2}} \xi(t)=\lambda \xi(t)+\zeta(t, \xi(t)), \quad \mathfrak{\Im}_{0^{+}}^{1-\gamma} \xi(0)=A, \\
& { }_{0^{+}}^{\mathbb{H}} \mathfrak{D}^{h, v ; g} \xi^{*}(t)=\lambda \xi^{*}(t)+\zeta\left(t, \xi^{*}(t)\right), \quad \mathfrak{\Im}_{0^{+}}^{1-\gamma} \xi^{*}(0)=A^{*},
\end{aligned}
$$


where the functions $\zeta(\cdot, \xi(\cdot)), \zeta\left(\cdot, \xi^{*}(\cdot)\right) \in C_{1-\gamma, g}([0, a])$ satisfy assumption (H1), for any $\xi, \xi^{*} \in C_{1-\gamma, g}([0, a])$.

Theorem 4. Assume that functions $\zeta$ and $\zeta^{*}$ satisfy assumption (H1). Let $\xi(t)$ and $\xi^{*}(t)$ be the solutions of problems (42), respectively. Then, we have the following estimate:

$$
\left|\xi(t)-\xi^{*}(t)\right| \leq \frac{\left|A-A^{*}\right|(g(t)-g(0))^{1-\gamma}}{\Gamma(\gamma)} \mathbb{E}_{h}\left((\lambda+L)[g(t)-g(0)]^{h}\right),
$$

for any $t \in[0, a]$.

Proof. Since $\xi(t)$ and $\xi^{*}(t)$ are the solutions of problems (42), respectively. For any $t \in[0, a]$, we have

$$
\begin{aligned}
\xi(t)= & \frac{(g(t)-g(0))^{\gamma-1}}{\Gamma(h)} A+\frac{\lambda}{\Gamma(h)} \int_{0}^{t} \mathfrak{Q}_{g}^{h}(t, s) \xi(s) \mathrm{d} s \\
& +\frac{1}{\Gamma(h)} \int_{0}^{t} \mathfrak{Q}_{g}^{h}(t, s) \zeta(s, \xi(s)) \mathrm{d} s, \\
\xi^{*}(t)= & \frac{(g(t)-g(0))^{\gamma-1}}{\Gamma(h)} A^{*}+\frac{\lambda}{\Gamma(h)} \int_{0}^{t} \mathfrak{Q}_{g}^{h}(t, s) \xi^{*}(s) \mathrm{d} s \\
& +\frac{1}{\Gamma(h)} \int_{0}^{t} \mathfrak{Q}_{g}^{h}(t, s) \zeta\left(s, \xi^{*}(s)\right) \mathrm{d} s .
\end{aligned}
$$

Using assumption (H1) and for any $t \in[0, a]$, we have the following estimate:

$$
\begin{aligned}
& \left|\xi(t)-\xi^{*}(t)\right| \leq \frac{\left|A-A^{*}\right|(g(t)-g(0))^{1-\gamma}}{\Gamma(\gamma)}+\frac{\lambda}{\Gamma(h)} \int_{0}^{t} \mathfrak{Q}_{g}^{h}(t, s)\left|\xi(s)-\xi^{*}(s)\right| \mathrm{d} s \\
& +\frac{1}{\Gamma(h)} \int_{0}^{t} \mathfrak{Q}_{g}^{h}(t, s)\left|\zeta(s, \xi(s))-\zeta\left(s, \xi^{*}(s)\right)\right| \mathrm{d} s \\
& \leq \frac{\left|A-A^{*}\right|(g(t)-g(0))^{1-\gamma}}{\Gamma(\gamma)}+\frac{(\lambda+L)}{\Gamma(h)} \int_{0}^{t} \mathfrak{Q}_{g}^{h}(t, s)\left|\xi(s)-\xi^{*}(s)\right| \mathrm{d} s . \\
& |\xi(t)| \leq\left(\frac{|A|(g(t)-g(0))^{1-\gamma}}{\Gamma(\gamma)}+\frac{\widehat{\zeta}(g(t)-g(0))^{h}}{\Gamma(1+h)}\right) \\
& \times \mathbb{E}_{h}\left((\lambda+L)[g(t)-g(0)]^{h}\right), \quad \forall t \in[0, a],
\end{aligned}
$$

If we put

$$
\begin{aligned}
& \widetilde{v}(t):=\left|\xi(t)-\xi^{*}(t)\right|, \\
& \widetilde{u}(t):=\frac{\left|A-A^{*}\right|(g(t)-g(0))^{1-\gamma}}{\Gamma(\gamma)}, \\
& \widetilde{g}(t):=\frac{(\lambda+L)}{\Gamma(h)},
\end{aligned}
$$

where $\widehat{\zeta}=\max _{s \in[0, a]} \zeta(s, 0)$.

for any $t \in[0, a]$, then inequality (45) becomes

$$
\widetilde{v}(t) \leq \widetilde{u}(t)+\widetilde{g}(t) \int_{0}^{t} \mathfrak{Q}_{g}^{h}(t, s) \widetilde{v}(s) \mathrm{d} s .
$$

Applying Gronwall Lemma 1 to (47), we obtain

$$
\widetilde{v}(t) \leq \widetilde{u}(t) \mathbb{E}_{h}\left((\lambda+L)[g(t)-g(0)]^{h}\right) .
$$

This gives inequality (43).

Proof. Let $\xi$ be any solution of problems (2) and (3). Then, we have

$$
\begin{aligned}
\xi(t)= & \frac{(g(t)-g(0))^{\gamma-1}}{\Gamma(\gamma)} A+\frac{\lambda}{\Gamma(h)} \int_{0}^{t} \mathfrak{Q}_{g}^{h}(t, s) \xi(s) \mathrm{d} s \\
& +\frac{1}{\Gamma(h)} \int_{0}^{t} \mathfrak{Q}_{g}^{h}(t, s) \zeta(s, \xi(s)) \mathrm{d} s, \quad \forall t \in[0, a] .
\end{aligned}
$$

Theorem 5. Assume that assumption (H1) is satisfied. If $\xi$ is any solution of problems (2) and (3), then

Using assumption (H1) and for any $t \in[0, a]$, we have 


$$
\begin{aligned}
|\xi(t)| \leq & \frac{|A|(g(t)-g(0))^{1-\gamma}}{\Gamma(\gamma)}+\frac{\lambda}{\Gamma(h)} \int_{0}^{t} \mathfrak{Q}_{g}^{h}(t, s)|\xi(s)| \mathrm{d} s \\
& +\frac{1}{\Gamma(h)} \int_{0}^{t} \mathfrak{Q}_{g}^{h}(t, s)|\zeta(s, \xi(s))| \mathrm{d} s \leq \frac{|A|(g(t)-g(0))^{1-\gamma}}{\Gamma(\gamma)}+\frac{\lambda}{\Gamma(h)} \int_{0}^{t} \mathfrak{Q}_{g}^{h}(t, s)|\xi(s)| \mathrm{d} s \\
& +\frac{1}{\Gamma(h)} \int_{0}^{t} \mathfrak{Q}_{g}^{h}(t, s)|\zeta(s, \xi(s))-\zeta(s, 0)+\zeta(s, 0)| \mathrm{d} s \\
\leq & \frac{|A|(g(t)-g(0))^{1-\gamma}}{\Gamma(\gamma)}+\frac{(\lambda+L)}{\Gamma(h)} \int_{0}^{t} \mathfrak{Q}_{g}^{h}(t, s)|\xi(s)| \mathrm{d} s+\frac{1}{\Gamma(h)} \int_{0}^{t} \mathfrak{Q}_{g}^{h}(t, s)|\zeta(s, 0)| \mathrm{d} s \\
\leq & \frac{|A|(g(t)-g(0))^{1-\gamma}}{\Gamma(\gamma)}+\frac{\widehat{\zeta}(g(t)-g(0))^{h}}{\Gamma(1+h)}+\frac{(\lambda+L)}{\Gamma(h)} \int_{0}^{t} \mathfrak{Q}_{g}^{h}(t, s)|\xi(s)| \mathrm{d} s .
\end{aligned}
$$

We put

$$
\begin{aligned}
& v(t):=\frac{|A|(g(t)-g(0))^{1-\gamma}}{\Gamma(\gamma)}+\frac{\widehat{\zeta}(g(t)-g(0))^{h}}{\Gamma(1+h)}, \\
& u(t):=\frac{(\lambda+L)}{\Gamma(h)},
\end{aligned}
$$

for any $t \in[0, a]$, which leads to the following estimate:

$$
|\xi(t)| \leq v(t)+u(t) \int_{0}^{t} \mathfrak{Q}_{g}^{h}(t, s)|\xi(s)| \mathrm{d} s, \quad \forall t \in[0, a] .
$$

Applying Gronwall Lemma 1, we obtain $|\xi(t)| \leq v(t) \mathbb{E}_{h}\left(u(t) \Gamma(h)[g(t)-g(0)]^{h}\right), \quad \forall t \in[0, a]$.

The proof is completed.
3.3. Hyers-Ulam Stability and Hyers-Ulam-Rassias Stability for Problem (2)

Theorem 6. Assume that assumption (H1) and (36) are satisfied. Then, problem (2) is Hyers-Ulam stable.

Proof. Let $\xi(t) \in C_{1-\gamma, g}([0, a])$ be a solution of (12) and let $\widehat{\xi}(t) \in C_{1-\gamma, g}([0, a])$ be a unique solution of (2). Then, for any, we have

$$
\begin{aligned}
\widehat{\xi}(t)= & \frac{(g(t)-g(0))^{\gamma-1}}{\Gamma(\gamma)} A+\frac{\lambda}{\Gamma(h)} \int_{0}^{t} \mathfrak{Q}_{g}^{h}(t, s) \widehat{\xi}(s) \mathrm{d} s \\
& +\frac{1}{\Gamma(h)} \int_{0}^{t} \mathfrak{Q}_{g}^{h}(t, s) \zeta(s, \widehat{\xi}(s)) \mathrm{d} s, \quad \forall t \in[0, a] .
\end{aligned}
$$

For any $t \in[0, a]$, we have

$$
\begin{aligned}
\mid \xi( & t)-\widehat{\xi}(t) \mid \\
= & \left|\xi(t)-\frac{(g(t)-g(0))^{\gamma-1}}{\Gamma(\gamma)} A-\frac{\lambda}{\Gamma(h)} \int_{0}^{t} \mathfrak{Q}_{g}^{h}(t, s) \widehat{\xi}(s) \mathrm{d} s-\frac{1}{\Gamma(h)} \int_{0}^{t} \mathfrak{Q}_{g}^{h}(t, s) \zeta(s, \widehat{\xi}(s)) \mathrm{d} s\right| \\
\leq & \left|\xi(t)-\frac{(g(t)-g(0))^{\gamma-1}}{\Gamma(\gamma)} A-\frac{\lambda}{\Gamma(h)} \int_{0}^{t} \mathfrak{Q}_{g}^{h}(t, s) \widehat{\xi}(s) \mathrm{d} s-\frac{1}{\Gamma(h)} \int_{0}^{t} \mathfrak{Q}_{g}^{h}(t, s) \zeta(s, \widehat{\xi}(s)) \mathrm{d} s\right| \\
& +\left|\frac{\lambda}{\Gamma(h)} \int_{0}^{t} \mathfrak{Q}_{g}^{h}(t, s) \widehat{\xi}(s) \mathrm{d} s+\frac{\lambda}{\Gamma(h)} \int_{0}^{t} \mathfrak{Q}_{g}^{h}(t, s) \zeta(s, \xi(s)) \mathrm{d} s-\frac{\lambda}{\Gamma(h)} \int_{0}^{t} \mathfrak{Q}_{g}^{h}(t, s) \widehat{\xi}(s) \mathrm{d} s-\frac{\lambda}{\Gamma(h)} \int_{0}^{t} \mathfrak{Q}_{g}^{h}(t, s) \zeta(s, \xi(s)) \mathrm{d} s\right| \\
\leq & \left|\xi(t)-\frac{(g(t)-g(0))^{\gamma-1}}{\Gamma(\gamma)} A-\frac{\lambda}{\Gamma(h)} \int_{0}^{t} \mathfrak{Q}_{g}^{h}(t, s) \widehat{\xi}(s) \mathrm{d} s-\frac{1}{\Gamma(h)} \int_{0}^{t} \mathfrak{Q}_{g}^{h}(t, s) \zeta(s, \widehat{\xi}(s)) \mathrm{d} s\right| \\
& +\frac{1}{\Gamma(h)} \int_{0}^{t} \mathfrak{Q}_{g}^{h}(t, s)|\xi(s)-\widehat{\xi}(t)| \mathrm{d} s+\frac{1}{\Gamma(h)} \int_{0}^{t} \mathfrak{Q}_{g}^{h}(t, s)|\zeta(s, \xi(s))-\zeta(s, \widehat{\xi}(s))| \mathrm{d} s \\
= & \left|\xi(t)-\frac{(g(t)-g(0))^{\gamma-1}}{\Gamma(\gamma)} A-\frac{\lambda}{\Gamma(h)} \int_{0}^{t} \mathfrak{Q}_{g}^{h}(t, s) \widehat{\xi}(s) \mathrm{d} s-\frac{1}{\Gamma(h)} \int_{0}^{t} \mathfrak{Q}_{g}^{h}(t, s) \zeta(s, \widehat{\xi}(s)) \mathrm{d} s\right| \\
& +\frac{(\lambda+L)}{\Gamma(h)} \int_{0}^{t} \mathfrak{Q}_{g}^{h}(t, s)|\xi(s)-\widehat{\xi}(t)| \mathrm{d} s .
\end{aligned}
$$


Moreover, due to the function $\xi(t)$ which satisfies inequality (12), there exists a function $h:[0, a] \longrightarrow \Re$ such that $|h(t)| \leq \mathcal{E}$, for any $t \in[0, a]$ and

$$
{ }_{0^{+}}^{\mathbb{H}} \mathfrak{D}^{h, v ; g} \xi(t)-\lambda \xi(t)-\zeta(t, \xi(t))=h(t), \quad \forall t \in[0, a] .
$$

Applying the fractional integral $\mathfrak{\Im}_{0^{+}}^{h ; g}(\cdot)$ to both sides of equation (57) and by using Theorem 1 , we obtain

$$
\mathfrak{\Im}_{0^{+}}^{h ; g}\left(\begin{array}{l}
\mathbb{1} \\
0^{+}
\end{array} \mathfrak{D}^{h, v ; g} \xi(t)-\lambda \xi(t)-\zeta(t, \xi(t))\right)=\mathfrak{\Im}_{0^{+}}^{h ; g} h(t), \quad \forall t \in[0, a] .
$$

Thus, we have

$$
\begin{aligned}
& \mid \xi(t)-\frac{(g(t)-g(0))^{\gamma-1}}{\Gamma(\gamma)} A-\frac{\lambda}{\Gamma(h)} \int_{0}^{t} \mathfrak{Q}_{g}^{h}(t, s) \xi(s) \mathrm{d} s \\
& -\frac{1}{\Gamma(h)} \int_{0}^{t} \mathfrak{Q}_{g}^{h}(t, s) \zeta(s, \xi(s)) \mathrm{d} s\left|\leq \mathfrak{J}_{0^{+}}^{h ; g}\right| h(t) \mid \\
& \leq \mathfrak{I}_{0^{+}}^{h ; g} \in=\frac{(g(t)-g(0))^{h}}{\Gamma(h)} \epsilon, \quad \forall t \in[0, a] .
\end{aligned}
$$
obtain

$$
\begin{aligned}
|\xi(t)-\widehat{\xi}(t)| \leq & \frac{(g(t)-g(0))^{h}}{\Gamma(h)} \epsilon+\frac{(\lambda+L)}{\Gamma(h)} \int_{0}^{t} \mathfrak{Q}_{g}^{h}(t, s) \mid \xi(s) \\
& -\widehat{\xi}(t) \mid \mathrm{d} s, \quad \forall t \in[0, a] .
\end{aligned}
$$

Applying Gronwall Lemma 1 to (60), we obtain

$$
\begin{aligned}
|\xi(t)-\hat{\xi}(t)| & \leq \frac{(g(t)-g(0))^{h}}{\Gamma(h)} \mathbb{E}_{h}\left((\lambda+L)[g(t)-g(0)]^{h}\right) \epsilon \\
& \leq \frac{(g(a)-g(0))^{h}}{\Gamma(h)} \mathbb{E}_{h}\left((\lambda+L)[g(t)-g(0)]^{h}\right) \epsilon \\
& \leq C_{\zeta} \epsilon, \quad \forall t \in[0, a],
\end{aligned}
$$

where $C_{\zeta}:=(g(a)-g(0))^{h} / \Gamma(h) \mathbb{E}_{h}\left((\lambda+L)[g(t)-g(0)]^{h}\right)$.

Based on the inequality above and Definition 2, we infer that problem (2) is Hyers-Ulam stable. The proof is completed.

Theorem 7. Assume that assumption (H1) and (36) are satisfied. If there exists function $\phi \in C([0, a], \Re)$ and the positive constant $C_{\phi}$ such that

$$
\frac{1}{\Gamma(h)} \int_{0}^{t} \mathfrak{Q}_{g}^{h}(t, s) \phi(s) \mathrm{d} s \leq C_{\phi} \phi(t), \quad \forall t \in[0, a],
$$

then problem (2) is Hyers-Ulam-Rassias stable.

Proof. Let $\xi(t) \in C_{1-\gamma, g}([0, a])$ be a solution of (12) and let $\widehat{\xi}(t) \in C_{1-\gamma, g}([0, a])$ be a unique solution of (2). Performing the same calculations as in Theorem 3, we have the estimation as follows:

$$
\begin{aligned}
|\xi(t)-\widehat{\xi}(t)| \leq & \xi(t)-\frac{(g(t)-g(0))^{\gamma-1}}{\Gamma(\gamma)} A-\frac{\lambda}{\Gamma(h)} \\
& \cdot \int_{0}^{t} \mathfrak{Q}_{g}^{h}(t, s) \xi(s) \mathrm{d} s \\
& -\frac{1}{\Gamma(h)} \int_{0}^{t} \mathfrak{Q}_{g}^{h}(t, s) \zeta(s, \xi(s)) \mathrm{d} s \\
& +\frac{(\lambda+L)}{\Gamma(h)} \int_{0}^{t} \mathfrak{Q}_{g}^{h}(t, s)|\xi(s)-\widehat{\xi}(t)| \mathrm{d} s .
\end{aligned}
$$

On the contrary, applying the fractional integral $\mathfrak{\Im}_{0^{+}}^{h ; g}(\cdot)$ to both sides of inequality (14) and by Theorem 1 and assumptions (61), we obtain

$$
\begin{aligned}
& \mid \xi(t)-\frac{(g(t)-g(0))^{\gamma-1}}{\Gamma(\gamma)} A-\frac{\lambda}{\Gamma(h)} \int_{0}^{t} \mathfrak{Q}_{g}^{h}(t, s) \xi(s) \mathrm{d} s \\
& -\frac{1}{\Gamma(h)} \int_{0}^{t} \mathfrak{Q}_{g}^{h}(t, s) \zeta(s, \xi(s)) \mathrm{d} s \mid \\
& \quad \leq \epsilon \mathfrak{J}_{0^{+}}^{h ; g} \phi(t) \leq C_{\phi} \phi(t) \epsilon, \quad \forall t \in[0, a] .
\end{aligned}
$$

From estimations (63) and (64), we imply

$$
|\xi(t)-\widehat{\xi}(t)| \leq C_{\phi} \phi(t) \epsilon+\frac{(\lambda+L)}{\Gamma(h)} \int_{0}^{t} \mathfrak{Q}_{g}^{h}(t, s)|\xi(s)-\widehat{\xi}(t)| \mathrm{d} s,
$$

Using Gronwall Lemma 1 and (64), we obtain

$$
\begin{aligned}
|\xi(t)-\widehat{\xi}(t)| \leq & C_{\phi} \mathbb{E}_{h}\left((\lambda+L)[g(t)-g(0)]^{h}\right) \phi(t) \epsilon \\
& \leq C_{\zeta, \phi} \epsilon, \quad \forall t \in[0, a],
\end{aligned}
$$

where $C_{\zeta, \phi}:=C_{\phi} \mathbb{E}_{h}\left((\lambda+L)[g(t)-g(0)]^{h}\right)$.

Similarly, based on the inequality above and Definition 2 , we infer that problem (2) is Hyers-Ulam-Rassias stable. The proof is completed.

3.4. Example. Let us consider the following problem:

$$
{ }_{0^{+}}^{\mathbb{H}} \mathfrak{D}^{h, v ; g} \xi(t)=\frac{1}{3} \xi(t)+\frac{1+\xi(t)}{9+e^{t}}, \quad \forall t \in[0,1 / 3],
$$

with the initial condition $\mathfrak{I}_{0^{+}}^{1-\gamma, g} \xi(0)=0$.

For this example, we only consider the two situation as below. The other one is considered similarly.

Situation 1. Let $g(t)=t$, taking the limit $v \longrightarrow 1$ on both sides of (8). Then, by Sousa et al. [16], we have

$$
{ }_{0^{+}}^{\mathbb{H}} \mathfrak{D}^{h, v ; g} \xi(t)==_{0^{+}}^{C} \mathfrak{D}^{h} \xi(t), \quad \forall t \in[0,1] .
$$


Combining (67) with (68), we infer that

$$
{ }_{0^{+}}^{C} \mathfrak{D}^{h} \xi(t)=\frac{1}{3} \xi(t)+\frac{1+\xi(t)}{9+e^{t}}, \quad \forall t \in[0,1 / 3],
$$

with the initial condition $\xi(0)=0$.

We consider $h=1 / 2, \lambda=1 / 3$, and

$$
\zeta(t, \xi(t)):=\frac{1+\xi(t)}{9+e^{t}}, \quad \forall t \in[0,1 / 3] .
$$

It is easy to see that $\zeta$ is a continuous function and it satisfies assumption (H1) with Lipschitz constant $L=1 / 10$. Indeed, for any $\xi_{1}, \xi \in C([0, a], \mathfrak{R})$, we have the following estimate:

$$
\begin{aligned}
\left|\zeta\left(t, \xi_{1}(t)\right)-\zeta\left(t, \xi_{2}(t)\right)\right| & =\left|\frac{1+\xi_{1}(t)}{9+e^{t}}-\frac{1+\xi_{2}(t)}{9+e^{t}}\right| \\
& \leq \frac{1}{9+e^{t}}\left|\xi_{1}(t)-\xi_{2}(t)\right| \\
& \leq \frac{1}{10}\left|\xi_{1}(t)-\xi_{2}(t)\right|, \quad \forall t \in[0,1 / 3] .
\end{aligned}
$$

Moreover, we have

$$
\begin{aligned}
& \frac{B(h, \gamma)(g(\tilde{a})-g(0))^{h}(\lambda+L)}{\Gamma(h)} \\
& \quad=\frac{B(1 / 2,1 / 2)(1 / 3-0)^{1 / 2}(1 / 3+1 / 10)}{\Gamma(1 / 2)} \approx 0.45<1 .
\end{aligned}
$$

We see that all the assumptions of Theorem 2 is satisfied. So, we infer that problem (69) has a unique solution on $[0,1 / 3]$.

Situation 2. Let $h=2 / 3, v=1 / 2$, and $g(t)=t^{\rho}, \rho>0$, for any $t \in[0,1 / 3]$. Then, by Sousa et al. [16], we have

$$
{ }_{0^{+}}^{\rho} \mathfrak{D}^{(2 / 3),(1 / 2)} \xi(t)=\frac{1}{3} \xi(t)+\frac{1+\xi(t)}{9+e^{t}}, \quad \forall t \in[0,1 / 3],
$$

with the initial condition $\rho \mathfrak{\Im}_{0^{+}}^{5 / 6} \xi(0)=0$.

Performing the same calculations as in Case 1, then it is also easy to check that problem (73) has a unique solution on $[0,1 / 3]$.

We put $\phi(t)=t^{3 \rho / 2-1}$ for any $t \in[0,1 / 3]$. Now, we will prove that problem (73) is Hyers-Ulam-Rassias stable. For any $t \in[0,1 / 3]$, we have

$$
\begin{aligned}
\frac{1}{\Gamma(h)} \int_{0}^{t} \mathfrak{Q}_{g}^{h}(t, s) \phi(s) \mathrm{d} s & =\frac{\rho^{1 / 3}}{\Gamma(2 / 3)} \int_{0}^{t} s^{-\rho / 2}\left(t^{\rho}-s^{\rho}\right)^{-1 / 3} \phi(s) \mathrm{d} s \\
& =\frac{\rho^{1 / 3}}{\Gamma(2 / 3)} \int_{0}^{t} s^{-\rho / 2}\left(t^{\rho}-s^{\rho}\right)^{-1 / 3} s^{3 \rho / 2-1} \mathrm{~d} s \\
& =\frac{\rho^{1 / 3}}{\Gamma(2 / 3)} \int_{0}^{t}\left(t^{\rho}-s^{\rho}\right)^{-1 / 3} s^{\rho-1} \mathrm{~d} s \\
& \leq \frac{3 \rho^{-2 / 3}}{2 \Gamma(2 / 3)}\left(\frac{1}{3}\right)^{1-5 \rho / 6} \phi(t), \quad \forall t \in[0,1 / 3] .
\end{aligned}
$$

Hence, assumption (73) of Theorem 3 is satisfied by $C_{\phi}:=3 \rho^{-2 / 3} / 2 \Gamma(2 / 3)(1 / 3)^{1-5 \rho / 6}>0$ with $\rho>0$. All the assumptions of Theorem 3 are satisfied. So, we imply problem (73) is Hyers-Ulam-Rassias stable.

\section{Data Availability}

No data were used to support this study.

\section{Conflicts of Interest}

The authors declare that they have no conflicts of interest.

\section{References}

[1] V. Daftardar-Gejji, Trends in Mathematics, Springer International Publishing, New York, NY, USA, 1st edition, 2019.

[2] A. A. Kilbas, H. M. Srivastava, and J. J. Trujillo, Theory and Applications of Fractional Differential Equations, Elsevier, New York, NY, USA, 1st edition, 2006.

[3] K. S. Miller and B. Ross, An Introduction to the Fractional Calculus and Fractional Differential Equations, Wiley, New York, NY, USA, 1993.

[4] M. Gaston, Developments in Mathematics, Vol. 27, SpringerVerlag, New York, NY, USA, 2012.

[5] Y. Zhou, J. Wang, and Lu Zhang, Basic Theory of Fractional Differential Equations, World Scientific, New York, NY, USA, 2nd edition, 2017.

[6] T. Jin, Y. Sun, and Y. Zhu, "Extreme values for solution to uncertain fractional differential equation and application to American option pricing model," Physica A: Statistical Mechanics and Its Applications, vol. 534, p. 122357, 2019.

[7] W. Ma, M. Jin, Y. Liu, and X. Xu, "Empirical analysis of fractional differential equations model for relationship between enterprise management and financial performance," Chaos, Solitons \& Fractals, vol. 125, pp. 17-23, 2019.

[8] P. Agarwal, S. Jain, and T. Mansour, "Further extended Caputo fractional derivative operator and its applications," Russian Journal of Mathematical Physics, vol. 24, no. 4, pp. 415-425, October 2017.

[9] P. Agarwal and R. Singh, "Modelling of transmission dynamics of Nipah virus (niv): a fractional order approach," Physica A: Statistical Mechanics and Its Applications, vol. 547, p. 124243, 2020.

[10] R. Ramesh, S. Harikrishnan, J. J. Nieto, and P. Prakash, "Oscillation of time fractional vector diffusion-wave equation with fractional damping," Opuscula Mathematica, vol. 40, no. 2, pp. 291-305, 2020.

[11] V. Deshmukh, K. Kanagarajan, and E. M. Elsayed, "Some existence and stability results for Hilfer-fractional implicit differential equations with nonlocal conditions," Mediterranean Journal of Mathematics, vol. 15, 2018.

[12] D. O’Regan and H. Van, “An initial value problem involving caputo-hadamard fractional derivative: the extremal solutions and stabilization," Mediterranean Journal of Mathematics Volume, vol. 4, no. 2, pp. 149-161, 2020.

[13] N. V. Hoa, "On the stability of fractional differential equations involving generalized Caputo fractional derivative," Mathematical Problems in Engineering, vol. 16, 2020.

[14] S. Tate and H. T. Dinde, "Some theorems on cauchy problem for nonlinear fractional differential equations with positive constant coefficient," Mediterranean Journal of Mathematics Volume, vol. 14, no. 72, 2017. 
[15] S. Tate, V. V. Kharat, and H. T. Dinde, "On nonlinear fractional integro-differential equations with positive constant coefficient," Mediterranean Journal of Mathematics Volume, vol. 16, no. 41, 2019.

[16] J. V. d. C. Sousa and E. Capelas de Oliveira, "On the $\psi$-Hilfer fractional derivative," Communications in Nonlinear Science and Numerical Simulation, vol. 60, pp. 72-91, 2018.

[17] J. V. d. C. Sousa and E. Capelas de Oliveira, "A Gronwall inequality and the Cauchy-type problem by means of $\psi$-Hilfer operator," Differential Equations \& Applications, vol. 11, no. 1, pp. 87-106, 2019.

[18] J. V. d. C. Sousa and E. Capelas de Oliveira, "On the fractional functional differential equation with abstract Volterra operator," Differential Equations and Dynamical Systems, vol. 60, 2019.

[19] J. V. d. C. Sousa, E. C. de Oliveira, and K. D. Kucche, "On the fractional functional differential equation with abstract Volterra operator," Bulletin of the Brazilian Mathematical Society, New Series, vol. 50, no. 4, pp. 803-822, 2019.

[20] J. V. d. C. Sousa, E. Capelas de Oliveira, E. Capelas de Oliveira, and F. G. Rodrigues, "Ulam-Hyers stabilities of fractional functional differential equations," AIMS Mathematics, vol. 5, no. 2, pp. 1346-1358, 2020.

[21] J. V. d. C. Sousa, K. D. Kucche, and E. C. de Oliveira, "Stability of $\psi$-Hilfer impulsive fractional differential equations," $A p$ plied Mathematics Letters, vol. 88, pp. 73-80, 2019.

[22] J. V. d. C. Sousa, F. G. Rodrigues, and E. Capelas de Oliveira, "Stability of the fractional Volterra integro-differential equation by means of $\psi$-Hilfer operator," Mathematical Methods In the Applied Sciences, vol. 42, no. 9, pp. 3033-3043, 2019.

[23] J. P. Kharade and K. D. Kucche, "On the impulsive implicit $\psi$-Hilfer fractional differential equations with delay," Mathematical Methods in the Applied Sciences, vol. 43, no. 4, p. 1938, 1952. 